\title{
Comparación de los criterios qSOFA, NEWS2 e Î́ndice de Choque más Lactato para la identificación temprana de pacientes con sepsis y choque séptico en el servicio de urgencias del Hospital General No. 450
}

\author{
Comparison of the qSOFA, NEWS2 and Lactate Shock Index criteria for the early \\ identification of patients with sepsis and septic shock in the emergency department of \\ General Hospital No. 450
}

\author{
Luisa F. Carbonell-Montes ${ }^{1 *}$, Maria del R Ramirez-Galindo ${ }^{1}$ y Fernando Vazquez-Alaniz ${ }^{2}$ \\ ${ }^{1}$ Servicio de Urgencias Adultos; ${ }^{2}$ Unidad de Investigación. Hospital General 450, Servicios de Salud de Durango, Durango, Durango, México
}

\section{Introducción}

La sepsis se define como la respuesta descontrolada del huésped frente a la infección, representa el 10\% de los ingresos a la unidad de cuidados intensivos y corresponde al $10-20 \%$ de la mortalidad hospitalaria'. Se observa con alta frecuencia en los servicios de urgencias y hoy día constituye una de las mayores causas de morbilidad y mortalidad ${ }^{2}$. Existen diferentes escalas para evaluar el pronóstico y el riesgo de muerte en los pacientes con diagnósticos de sepsis y choque séptico, que resultan poco útiles para el diagnóstico inicial. No hay una prueba de referencia para diagnosticar este síndrome y, por tanto, existe la necesidad de establecer una escala fácil y rápida de aplicar en el servicio de urgencias $^{3}$. En este estudio se propuso una escala compuesta por el índice de choque, relación entre la frecuencia cardiaca y la presión arterial sistólica, un parámetro fácil de calcular, objetivo y que refleja el estado hemodinámico de la macrocirculación, más la determinación del lactato, un marcador del estado microcirculatorio que refleja el grado de hipoperfusión tisular secundario al proceso infeccioso, de tal manera que al unir ambos parámetros se obtiene una idea del estado general del paciente con sepsis y choque séptico.

El objetivo de este estudio fue comparar la sensibilidad y la especificidad de las escalas qSOFA (quick Sequential Organ Failure Assessment), NEWS2 (National Early Warning Score 2) y LalC (Lactato más Índice de Choque) para la identificación temprana de pacientes con sepsis y choque séptico en el servicio de urgencias del Hospital General No. 450.

\section{Método}

El protocolo fue evaluado y aprobado por el comité de ética en investigación del Hospital General No. 450. Se realizó un estudio descriptivo, prospectivo, transversal y comparativo que incluyó los pacientes que ingresaron a urgencias con dos o más criterios de síndrome de respuesta inflamatoria sistémica; se excluyeron aquellos con diagnóstico de ingreso de traumatismo, insuficiencia hepática y sangrado. Se aplicaron las escalas qSOFA, NEWS2 y LalC para comparar su sensibilidad y especificidad en el diagnóstico de sepsis y choque séptico.

\section{Correspondencia:}

*Luisa F. Carbonell-Montes

E-mail: luisafernandacarbonell@ @otmail.com
Disponible en internet: 07-03-2022

Fecha de recepción: 29-11-2021

Fecha de aceptación: 21-12-2021 DOI: 10.24875/REIE.21000204
Rev Educ Investig Emer. 2022;4(Supl 1):48-50 www.medicinadeemergencias.com bajo la licencia CC BY-NC-ND (http://creativecommons.org/licenses/by-nc-nd/4.0/). 


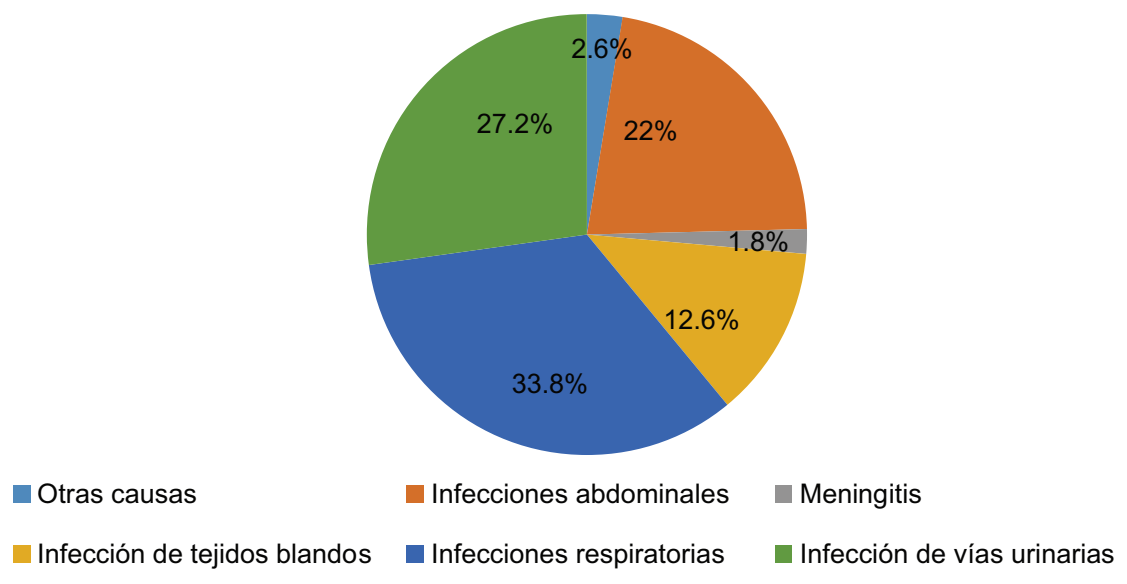

Figura 1. Principales causas de sepsis.

Tabla 1. Descripción demográfica y clínica de la población

\section{Total pacientes: 358}

\begin{tabular}{|l|c|}
\hline $\begin{array}{l}\text { Sexo } \\
\text { Femenino } \\
\text { Masculino }\end{array}$ & $171(47.7 \%)$ \\
\hline Edad media (mínima-máxima) & $187(52.3 \%)$ \\
\hline Origen de la sepsis & $56(18-93)$ años \\
Infección de vías respiratorias & \\
Infección de vías urinarias & $91(33.8 \%)$ \\
\hline Infección de tejidos blandos & $73(27.2 \%)$ \\
Meningitis & $34(12.6 \%)$ \\
\hline Infecciones abdominales & $5(1.8 \%)$ \\
Otras & $59(22 \%)$ \\
\hline qSOFA & $7(2.6 \%)$ \\
Sin riesgo de sepsis & \\
Riesgo bajo de sepsis & $22(6.1 \%)$ \\
Riesgo moderado de sepsis & $132(36.8 \%)$ \\
\hline Riesgo alto de sepsis & $141(39.3 \%)$ \\
\hline NEWS2 & $63(17.5 \%)$ \\
\hline Riesgo bajo de sepsis & \\
\hline Riesgo moderado de sepsis & $81(22.6 \%)$ \\
Riesgo alto de sepsis & $75(20.9 \%)$ \\
\hline LalC & $202(56.3 \%)$ \\
\hline Sin riesgo de sepsis & \\
\hline Riesgo bajo de sepsis & $9(2.5 \%)$ \\
\hline Riesgo moderado de sepsis & $70(19.5 \%)$ \\
Riesgo alto de sepsis & $116(32.3 \%)$ \\
\hline
\end{tabular}

\section{Resultados}

Se evaluaron 358 pacientes que cumplieron los criterios de inclusión. De ellos, el $52.2 \%$ fueron de sexo masculino y el $47.8 \%$ de sexo femenino. El $24.9 \%$ de los pacientes no presentaron sepsis, mientras que el
$75.1 \%$ presentaron sepsis o choque séptico (o ambos). El $76 \%$ de las mujeres fueron diagnosticadas con sepsis o choque séptico, mientras que el $24 \%$ fueron negativas para sepsis y choque séptico. De la totalidad de los hombres estudiados, el $74.3 \%$ tuvieron diagnóstico de sepsis o choque séptico, mientras que el 25.7\% dieron negativo para sepsis (Tabla 1). La principal causa de sepsis fue la infección de vías respiratorias, en el $33.8 \%$ de los casos, seguida de la infección de vías urinarias, en el $27.2 \%$, y en menor proporción la infección de tejidos blandos, en el 12.6\% (Fig. 1).

Respecto a la sensibilidad y la especificidad de las escalas evaluadas, la curva ROC (Receiver Operating Characteristic) muestra que, de las tres escalas evaluadas, la qSOFA tiene las menores sensibilidad y especificidad, con un $61.9 \%$ y un $48.1 \%$, respectivamente; la escala NEWS2 mostró una sensibilidad del $64.4 \%$ y una especificidad del $50.6 \%$, mientras que la escala LalC obtuvo una sensibilidad del $68.6 \%$ y una especificidad del $55.1 \%$ (Fig. 2).

\section{Discusión}

La sepsis y el choque séptico son problemas de salud importantes, que afectan a millones de personas en todo el mundo y causan la muerte de uno de cada tres afectados ${ }^{4}$. La identificación temprana y el manejo apropiado en las primeras horas después del desarrollo de la sepsis mejoran los resultados ${ }^{5}$. Con el objetivo de promover la identificación temprana de la sepsis se han utilizado variables clínicas y herramientas de cribado, como los signos vitales, los signos de infección y las escalas qSOFA y NEWS) ${ }^{6}$, todas ellas con una amplia variación en 


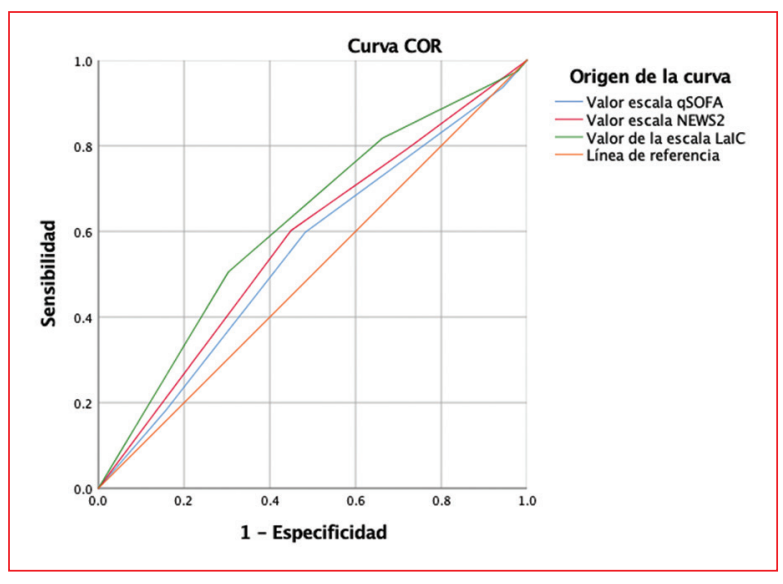

Figura 2. La curva COR muestra que la qSOFA tuvo una sensibilidad del $61.9 \%$ y una especificidad del $48.1 \%$, la NEWS2 tuvo una sensibilidad del $64.4 \%$ y una especificidad del $50.6 \%$, y la LalC tuvo una sensibilidad del $68.6 \%$ y una especificidad del $55.1 \%$.

la precisión diagnóstica 7 . Parte de esta variabilidad diagnóstica radica en lo subjetivo de la qSOFA, ya que los tres valores que mide son dependientes del operador, y en el mayor tiempo que requiere la escala NEWS2, pues consta de ocho parámetros de evaluación sistémica. Por ello, se propuso la escala LaIC las unidades se definen como Unidades arbitrarias (UA) como método diagnóstico al ingreso de los pacientes al servicio de urgencias para el reconocimiento de aquellos con riesgo de sepsis y choque séptico, y resultó ser un poco más sensible y específica que la qSOFA y la NEWS2.

\section{Conclusiones}

Aunque la sensibilidad y la especificidad de la escala LaIC también son bajas, ofrece un mayor acercamiento al diagnóstico de sepsis. Por lo tanto, debido a su objetividad, disponibilidad y sencillez, se propone como otra alternativa eficaz y segura para mejorar el diagnóstico oportuno y el manejo dirigido, con impacto en la mortalidad por esta patología.

\section{Agradecimientos}

Al personal médico, de enfermería y de diagnóstico, que siempre está dispuesto a atender y salvaguardar la vida de los pacientes con sepsis y choque séptico que acuden a nuestra sala de urgencias.

\section{Financiamiento}

Los recursos de este estudio fueron proporcionados por los propios investigadores.

\section{Conflicto de intereses}

Los autores declaran que no tienen ningún conflicto de intereses.

\section{Responsabilidades éticas}

Protección de personas y animales. Los autores declaran que para esta investigación no se han realizado experimentos en seres humanos ni en animales.

Confidencialidad de los datos. Los autores declaran que en este artículo no aparecen datos de pacientes.

Derecho a la privacidad y consentimiento informado. Los autores declaran que en este artículo no aparecen datos de pacientes.

\section{Bibliografía}

1. Freund $Y$, Lemachatti N, Krastinova E, Van Laer M, Claessens YE, Avondo A, et al. Prognostic accuracy of Sepsis-3 criteria for in-hospital mortality among patients with suspected infection presenting to the emergency department. JAMA. 2017;317:301-8.

2. Redondo-González A, Varela-Patiño M, Álvarez-Manzanares J, Oliva-Ramos JR, López-Izquierdo R, Ramos-Sánchez C, et al. Valoración de escalas de gravedad en pacientes incluidos en un código sepsis en un servicio de urgencias hospitalario. Rev Esp Quimioter. 2018;31:316-22.

3. Carrillo Esper R, Peña Pérez CA, Sosa García JO. Sepsis. De las bases moleculares a la campaña para incrementar la supervivencia. México: Academia Nacional de Medicina; 2015. p. 30-8. Disponible en: https://www.anmm.org.mx/publicaciones/CAnivANM150/L31_ANM_ SEPSIS.pdf

4. Fleischmann C, Scherag A, Adhikari NK, Hartog CS, Tsaganos T, Schlattmann $\mathrm{P}$, et al.; International Forum of Acute Care Trialists. Assessment of global incidence and mortality of hospital-treated sepsis. Current estimates and limitations. Am J Respir Crit Care Med. 2016;193:259-72.

5. Evans L, Rhodes A, Alhazzani W, Antonelli M, Coopersmith CM, French C et al. Surviving sepsis campaign: international guidelines for management of sepsis and septic shock 2021. Intensive Care Med. 2021;47:1181-247.

6. Alberto L, Marshall AP, Walker R, Aitken LM. Screening for sepsis in general hospitalized patients: a systematic review. J Hosp Infect. 2017;96:305-15

7. Warttig S, Alderson P, Evans DJ, Lewis SR, Kourbeti IS, Smith AF. Automated monitoring compared to standard care for the early detection of sepsis in critically ill patients. Cochrane Database Syst Rev. 2018; (6):CD012404. 